\title{
An invariant variational principle for canonical flows on Lie groups
}

\author{
Zoltán Muzsnay ${ }^{\mathrm{a})}$ \\ Department of Mathematics, University of Debrecen, Debrecen, H-4032, Hungary
}

(Received 18 May 2005; accepted 19 September 2005)

In this paper we examine the existence of Lie groups, whose canonical geodesic flows are variational with respect to a left-invariant regular-but not necessarily quadratic (i.e., metric)—Lagrange function. We give effective necessary and sufficient conditions for the existence of an invariant variational principle generating the canonical flow. With these results, taken in conjunction with the classification of Lie algebras, we solve the inverse problem of invariant Lagrangian dynamics in dimensions up to four. (C) 2005 American Institute of Physics.

[DOI: $10.1063 / 1.2118487]$

\section{INTRODUCTION}

One of the basic objects of classical physics is the equation of motion of a system. In the most relevant cases it can be derived as the Euler-Lagrange equation of a regular Lagrange function. An old, and in the general case still unsolved problem, posed first in 1887 by Helmholtz, is the determination and classification of second order differential equations derivable from a variational principle. This is the inverse problem of the calculus of variations. Moreover, since the EulerLagrange equation inherits the symmetries of the Lagrangian, one can also consider the invariant version of the inverse problem of the calculus of variations, determine if an equation of motion possessing some symmetry property can be derived as the Euler-Lagrange equation of a regular Lagrangian having the same symmetry.

There are interesting examples of this phenomena in the cases of motions on Lie groups governed by the canonical symmetric, linear connections of the Lie group. ${ }^{1}$ We remark that the corresponding canonical flow occurs in the one-dimensional reductions of well-known models of theoretical physics, like the $\sigma$ or chiral models. One can say that all possible situations occur in these deceptively simple systems. This claim is illustrated by the following examples: (1) on a semisimple Lie group the canonical flow can be derived from the Lagrangian corresponding to the bi-invariant Killing metric of the Lie algebra. In this case the Lagrangian is obviously invariant; (2) on the Heisenberg group or on the affine group of the line the canonical flows are variational, but the Lagrangians are not invariant with respect to the natural action of the groups (see paragraph 5); (3) on the four-dimensional Lie groups $A_{4,7}, A_{4,9 b}$, and $A_{4,11 a}$ the canonical flows are not variational. $^{9}$

Moreover, one can investigate the unicity of the Lagrangian (up to an inessential total derivative term). The unicity problem and the existence of invariant variational principle might be related to each other in some cases, as if the equation of motion has some symmetry, but the Lagrangian does not possess that one, then the Lagrangian is necessarily nonunique, as a new Lagrangian obtained from the old one by a symmetry transformation must give the same EulerLagrange equation.

In this paper we examine the existence of Lie groups, other than the semisimple groups, where the geodesic flow associated to the canonical connection is variational with respect to a leftinvariant regular (but not necessarily quadratic, i.e., metric) Lagrange function. In this case we

\footnotetext{
a)Electronic mail: muzsnay@math.klte.hu
} 
will say that there exists a $G$-invariant variational principle for the canonical flow of the group $G$. We give an effective necessary and sufficient condition for the existence of a $G$-invariant variational principle for the canonical flow. Using this result, we determine the Lie groups up to four dimensions for which the canonical flow is variational with respect to a nondegenerate leftinvariant Lagrangian.

\section{INVERSE PROBLEM OF THE CALCULUS OF VARIATIONS ON LIE GROUPS}

On a Lie group $G$ there are three kinds of natural linear connections, the plus, minus, and zero connections introduced first by Cartan and Schouten. ${ }^{1}$ A simple way to define these connections is to give their values on left-invariant vector-fields $X, Y$ by

$$
\nabla_{X}^{+} Y=[X, Y], \quad \nabla_{X}^{-} Y=0, \quad \nabla_{X}^{0} Y=\frac{1}{2}[X, Y],
$$

and extend them to arbitrary vector fields by making them tensorial in the $X$ argument and satisfy the Leibniz rule in the $Y$ argument. The curvature tensors of the minus and plus connections are zero, but in general, the corresponding torsion tensors are nonvanishing. All three of the natural connections have the same geodesics. We shall denote $\nabla^{0}$ simply by $\nabla$ and refer to it as the canonical symmetric, linear connection.

Let $E: T G \rightarrow \mathbb{R}$ be a smooth function on the tangent bundle of a Lie group $G$, called the Lagrange function. For an arbitrary curve $g(t):\left[t_{0}, t_{1}\right] \rightarrow G$ one can consider the functional $I[g]$ $:=\int_{t_{0}}^{t_{1}} E(g(t), \dot{g}(t)) \mathrm{d} t$. A standard argument shows that the functions $g(t)$ giving extrema of this functional (in the class of variations preserving the endpoints) satisfy the Euler-Lagrange equations. In standard local coordinates $\left\{g^{i}\right\}$ on $G$ they are

$$
\frac{d}{\mathrm{~d} t}\left(\frac{\partial E}{\partial \dot{g}^{i}}\right)-\frac{\partial E}{\partial g^{i}}=0 .
$$

If the Lagrangian $E$ is regular, then (1) is a second order ordinary differential equation.

In a series of papers, Thompson and his co-workers investigated the inverse problem of Lagrangian dynamics for the geodesic flow associated to $\nabla$ (see Refs. 2, 7, and 9). Here one wishes to decide if there exists a Lagrangian function defined on an open subset of the tangent bundle of the Lie group whose Euler-Lagrange equations coincide with the geodesic equations of $\nabla$. In this paper we consider the invariant version of this problem. We examine the existence of Lie groups, whose canonical geodesic flows are variational with respect to a left-invariant regularbut not necessarily quadratic_Lagrange function.

\section{DIFFERENTIAL GEOMETRIC BACKGROUND OF THE INVERSE PROBLEM OF THE CALCULUS OF VARIATIONS}

We present here the basic objects that play a role in the theory. More details can be found in Refs. 5 and 7

If $M$ is a manifold $T M$ denotes its tangent space and $\pi$ the natural submersion. Let $J: T T M$ $\rightarrow T T M$ be the canonical vertical endomorphism, and let $C \in \mathfrak{X}(T M)$ be the Liouville vector field, if $\left(x^{\alpha}\right)$ is a local coordinate system on $M$ and $\left(x^{\alpha}, y^{\alpha}\right)$ is the induced coordinate system on $T M$, then

$$
J=\mathrm{d} x^{\alpha} \otimes \frac{\partial}{\partial y^{\alpha}}, \quad C=y^{\alpha} \frac{\partial}{\partial y^{\alpha}} .
$$

A (non-linear) connection on $M$ is a tensor field of type (1-1) $\Gamma$ on $T M$ such that $J \Gamma=J$ and $\Gamma J$ $=-J$ (see Ref. 4). If $\Gamma$ is a connection, then $\Gamma^{2}=i d_{T T M}$ and the eigenspace corresponding to the eigenvalue -1 is the vertical space $V_{z}$. Then, at any $z \in T M$, we have the splitting $T_{z} T M=H_{z}$ $\oplus V_{z}$, where $H_{z}$ is the eigenspace corresponding to +1 . The subspace $H_{z}$ is called the horizontal space. In the sequel we will write 


$$
h:=\frac{1}{2}(I+\Gamma), \quad v:=\frac{1}{2}(I-\Gamma),
$$

for the horizontal and vertical projectors.

Definition 1: A Lagrangian is a map E:TM $\rightarrow \mathbb{R}$ that is smooth on at least an open subset of $T M$. The Lagrangian $E$ is said to be regular if

$$
\operatorname{det}\left(\frac{\partial^{2} E}{\partial y^{\alpha} \partial y^{\beta}}\right) \neq 0
$$

The Lagrangian $E$ is regular if and only if the 2-form $\Omega_{E}:=\mathrm{d} d_{J} E$ has maximal rank. In this case, the vector field $S$ on $T M$ defined by the equation

$$
i_{S} \Omega_{E}=d\left(E-\mathcal{L}_{C} E\right)
$$

is a spray (that is $J S=C$ ), and the paths of $S$ are the solutions of the Euler-Lagrange equations, ${ }^{3}$

$$
\frac{d}{\mathrm{~d} t} \frac{\partial E}{\partial \dot{x}^{\alpha}}-\frac{\partial E}{\partial x^{\alpha}}=0, \quad \alpha=1, \ldots, n .
$$

Let us fix a second order differential equation, or in other words a spray $S$ on the manifold $M$. Then, to every Lagrangian $E$ a scalar 1-form $\omega_{E}$ can be associated by

$$
\omega_{E}:=i_{S} \Omega_{E}+\mathrm{d} \mathcal{L}_{C} E-\mathrm{d} E,
$$

which is called Euler-Lagrange form. According to Eq. (4) we have $\omega_{E} \equiv 0$ if and only if the path of the spray is the solution of the Euler-Lagrange equation (5) associated to E. (See Refs. 5 and 7.)

\section{LEFT-INVARIANT VARIATIONAL PRINCIPLE FOR THE CANONICAL CONNECTION}

In the sequel we will consider the case, where $M:=G$ is a Lie group. We will denote by $L_{x} g$ or simply by $x g$ the left translation of $g \in G$ by $x \in G$. Let $(x)$ be coordinates on $G$, and let $(x, y)$ be the standard associated coordinate system on $T G$. We will also use the "left invariant" coordinates $(x, \alpha)$ on $T G \simeq G \times \mathfrak{g}$, where $\alpha=\left(L_{x^{-1}}\right) * y$ is the Maurer-Cartan form. The corresponding coordinates on $T T G$ are $(x, \alpha, X, A)$, that is,

$$
(x, \alpha, X, A)=\left.X \frac{\partial}{\partial x}\right|_{(x, \alpha)}+\left.A \frac{\partial}{\partial \alpha}\right|_{(x, \alpha)} .
$$

Since the coordinates $\alpha=\left(\alpha_{i}\right)$ and $A=\left(A_{i}\right)$ are left-invariant coordinates, we find that the left translation by a group element $g$ induces on TTG the following action:

$$
L_{g}(x, \alpha, X, A)=(g x, \alpha, g X, A)=\left.g X \frac{\partial}{\partial x}\right|_{(g x, \alpha)}+\left.A \frac{\partial}{\partial \alpha}\right|_{(g x, \alpha)} .
$$

The canonical projection $\pi: T G \rightarrow G$ is $(x, \alpha) \rightarrow x$, therefore $\pi_{*}: T T G \rightarrow T G$ is given by $(x, \alpha, X, A) \rightarrow\left(x, x^{-1} X\right)$ and the vertical subspace on $(x, \alpha) \in T G$ is

$$
V_{(x, \alpha)} T G:=\operatorname{Ker} \pi_{*}=\{(x, \alpha, 0, b) \mid b \in \mathfrak{g}\} .
$$

Second order differential equations are described by vector-fields $S$ (called spray) on the tangent space, that have the characteristic property $J S=C$. On a Lie group the geodesic flow of the canonical connection is described by the system

$$
\ddot{x}=\dot{x} x^{-1} \dot{x},
$$

and the vector field on the tangent space, corresponding to the geodesic flow of the canonical connection is the spray $S$ where 


$$
S_{(x, \alpha)}=(x, \alpha, x \alpha, 0)=\left.x \alpha \frac{\partial}{\partial x}\right|_{(x, \alpha)} .
$$

Then $\gamma_{t}$ is a geodesic of $\nabla$ if and only if the equation $S_{\dot{\gamma}}=\ddot{\gamma}$ holds. The horizontal and vertical projections of the connection $\Gamma:=[J, S]$ associated the spray $S$ are $h$ and $v$ where

$$
\begin{gathered}
v(x, b, x a, c)=\left(x, b, 0, \frac{1}{2}[a, b]+c\right)=\left.\left(\frac{1}{2}[a, b]+c\right) \frac{\partial}{\partial \alpha}\right|_{(x, b)}, \\
h(x, b, x a, c)=\left(x, b, x a,-\frac{1}{2}[a, b]\right)=\left.x a \frac{\partial}{\partial x}\right|_{(x, b)}-\left.\frac{1}{2}[a, b] \frac{\partial}{\partial \alpha}\right|_{(x, b)},
\end{gathered}
$$

therefore the horizontal and vertical lifts of a left-invariant vector field $X=(x, a)$ are

$$
\begin{gathered}
X_{(x, \alpha)}^{h}=\left(x, \alpha, x a,-\frac{1}{2}[a, \alpha]\right)=\left.x a \frac{\partial}{\partial x}\right|_{(x, \alpha)}-\left.\frac{1}{2}[a, \alpha] \frac{\partial}{\partial \alpha}\right|_{(x, \alpha)}, \\
X_{(x, \alpha)}^{v}=(x, \alpha, 0, a)=\left.a \frac{\partial}{\partial \alpha}\right|_{(x, \alpha)} .
\end{gathered}
$$

Moreover, with $(x, \alpha)$ as a local coordinate system, the vertical endomorphism and the Liouville vector fields are

$$
J=\left(x^{-1} \mathrm{~d} x\right) \otimes \frac{\partial}{\partial \alpha}, \quad C=\alpha \frac{\partial}{\partial \alpha} .
$$

Indeed,

$$
\begin{gathered}
C_{(x, \alpha)}=\left.\frac{d}{\mathrm{~d} t}\right|_{t=0}\left(x, e^{t} \alpha\right)=(x, \alpha, 0, \alpha), \\
J(x, \alpha, A, B)=\left.\frac{d}{\mathrm{~d} t}\right|_{t=0}\left(x, \alpha+t x^{-1} A\right)=\left(x, \alpha, 0, x^{-1} A\right) .
\end{gathered}
$$

For more details see Ref. 7.

Let us examine the existence of a left-invariant variational principle for the canonical flow of Lie groups. We have the following.

Proposition 2: The canonical flow of the Lie group $G$ is variational with respect a leftinvariant Lagrangian if and only if there exists an ad-invariant function $\mathcal{E}: \mathfrak{g} \rightarrow \mathbb{R}$ with nondegenerate Hessian.

To prove the proposition, we compute the Euler-Lagrange equation for the canonical flow of $G$. Using the notation introduced in (6), the Euler-Lagrange partial differential equation associated to a spray $S$ can be written as

$$
\omega_{E} \equiv 0,
$$

where the unknown is the Lagrangian $E$. Let us compute explicitly the equation (11). If $X$ $=(x, a)$ denotes a left-invariant vector field on $G$ corresponding to $a \in \mathfrak{g}$, and $X^{v}, X^{h}$ are its vertical and horizontal lifts, then we have $\omega_{E}\left(X^{v}\right) \equiv 0$, since $\omega_{E}$ is semibasic. Moreover, we have 


$$
\begin{aligned}
\omega_{E}\left(X^{h}\right) & =\left(i_{S} \mathrm{~d} d_{J} E+\mathrm{d} \mathcal{L}_{C} E-\mathrm{d} E\right)\left(X^{h}\right)=\mathrm{d} d_{J} E\left(S, X^{h}\right)+\mathrm{d} \mathcal{L}_{C} E\left(X^{h}\right)-\mathrm{d} E\left(X^{h}\right) \\
& =S\left(J X^{h}(E)\right)-X^{h}(J S(E))-J\left[S, X^{h}\right] E+X^{h}(C(E))-X^{h} E \\
& =S\left(X^{v}(E)\right)-J\left[S, X^{h}\right] E-X^{h} E .
\end{aligned}
$$

Taking into account (8) and the local expression (9) of $X^{h}$ we have

$$
\begin{gathered}
{\left[X^{h}, S\right]=\left[x a \frac{\partial}{\partial x}-\frac{1}{2}[a, \alpha] \frac{\partial}{\partial \alpha}, x \alpha \frac{\partial}{\partial x}\right]=\frac{1}{2} x[a, \alpha] \frac{\partial}{\partial x},} \\
J\left[X^{h}, S\right]=\frac{1}{2} J\left(x[a, \alpha] \frac{\partial}{\partial x}\right)=\frac{1}{2}[a, \alpha] \frac{\partial}{\partial \alpha},
\end{gathered}
$$

and

$$
\begin{aligned}
\omega_{E}\left(X^{h}\right) & =x \alpha \frac{\partial}{\partial x}\left(a \frac{\partial}{\partial \alpha}(E)\right)-\frac{1}{2}[a, \alpha] \frac{\partial}{\partial \alpha}(E)-\left(x a \frac{\partial}{\partial x}-\frac{1}{2}[a, \alpha] \frac{\partial}{\partial \alpha}\right) E \\
& =x \alpha a \frac{\partial^{2} E}{\partial x \partial \alpha}+\frac{1}{2}[a, \alpha] \frac{\partial E}{\partial \alpha}-x a \frac{\partial E}{\partial x}+\frac{1}{2}[a, \alpha] \frac{\partial E}{\partial \alpha} \\
& =[a, \alpha] \frac{\partial E}{\partial \alpha}+x \alpha a \frac{\partial^{2} E}{\partial x \partial \alpha}-x a \frac{\partial E}{\partial x} .
\end{aligned}
$$

If the Lagrangian is left-invariant, then $\partial E / \partial x=0$ and we obtain that

$$
\omega\left(X^{h}\right)=[a, \alpha] \frac{\partial E}{\partial \alpha},
$$

consequently the canonical connection of the group $G$ is variational with respect to a left-invariant regular Lagrangian $E$ if and only if

$$
\begin{gathered}
\frac{\partial E}{\partial x}=0, \\
{[a, \alpha] \frac{\partial E}{\partial \alpha}=0, \quad \forall a \in \mathfrak{g},}
\end{gathered}
$$

where (12) expresses the fact that $E$ is left-invariant and (13) expresses that $E$ is a solution of the Euler-Lagrange equation (11). Therefore the canonical flow is variational with respect to an invariant Lagrangian if and only if the Frobenius differential system (12) and (13) has a solution $E: T G \simeq G \times \mathfrak{g} \rightarrow \mathbb{R}$ satisfying the regularity condition, or equivalently, there exists a function $\mathcal{E}: \mathfrak{g} \rightarrow \mathbb{R}$ satisfying the equation

$$
[a, \alpha] \frac{\partial \mathcal{E}}{\partial \alpha}=0,
$$

for all $a \in \mathfrak{g}$, such that the Hessian matrix $\left(\partial^{2} \mathcal{E} / \partial \alpha_{i} \partial \alpha_{j}\right)$ is nondegenerate. The equation (14) is identically satisfied if and only if $\mathcal{E}$ is constant on the orbit of the ad representation of $\mathfrak{g}$.

Let $\left\{e_{1}, \ldots, e_{n}\right\}$ be a basis of $\mathfrak{g}$. Then the structure constants $C_{\alpha \beta}^{\gamma}$ of the Lie algebra $\mathfrak{g}$ are defined by

$$
\left[e_{\alpha}, e_{\beta}\right]=C_{\alpha \beta}^{\gamma} e_{\gamma} .
$$

We denote by $\left(\alpha_{i}\right)$ the coordinate of $\alpha \in \mathfrak{g}$. We have the following. 
Theorem 3: There exists a left-invariant variational principle for the canonical flow of the Lie group $G$ in a neighborhood of a generic element $\alpha \in \mathfrak{g}$ if and only if the linear system

$$
\begin{gathered}
C_{i j}^{k} \alpha_{j} x_{k}=0, \quad i=1, \ldots, n, \\
C_{i j}^{k} x_{k}+C_{j m}^{k} \alpha_{m} x_{i k}=0, \quad i, j=1, \ldots, n,
\end{gathered}
$$

has a solution $\left\{x_{i}=\epsilon_{i}, x_{i j}=\epsilon_{i j}\right\}$ satisfying the condition $\operatorname{det}\left(\epsilon_{i j}\right) \neq 0$.

Proof: Let us consider the distribution $\Delta$ in the tangent space of $\mathfrak{g}$ defined as

$$
\Delta_{\alpha}:=\left\{X_{a}:=\left.[a, \alpha] \frac{\partial}{\partial \alpha}\right|_{\alpha} \mid a \in \mathfrak{g}\right\},
$$

for $\alpha \in \mathfrak{g}$. If $a$ and $b$ are two elements of the Lie algebra $\mathfrak{g}$, then

$$
\left[[a, \alpha] \frac{\partial}{\partial \alpha},[b, \alpha] \frac{\partial}{\partial \alpha}\right]=[[\alpha, a], b] \frac{\partial}{\partial \alpha}-[[\alpha, b], a] \frac{\partial}{\partial \alpha}=[\alpha,[a, b]] \frac{\partial}{\partial \alpha},
$$

that is for every $X_{a} \in \Delta$ and $X_{b} \in \Delta$ we have $\left[X_{a}, X_{b}\right]=X_{[a, b]} \in \Delta$. This shows that $\Delta$ is involutive and the system (14) is integrable.

Let us denote by $J_{1}(\mathfrak{g}, \mathbb{R})$ and by $J_{2}(\mathfrak{g}, \mathbb{R})$ the first and second order jet spaces of real valued functions on $\mathfrak{g}$. As usual, a local coordinates system on $J_{1}(\mathfrak{g}, \mathbb{R})$ and $J_{2}(\mathfrak{g}, \mathbb{R})$ can be given as follows: if $\mathcal{E}: \mathfrak{g} \rightarrow \mathrm{R}$, then

$$
j_{1, \alpha}(\mathcal{E})=\left(\alpha, \epsilon, \epsilon_{i}\right), \quad j_{2, \alpha}(\mathcal{E})=\left(\alpha, \epsilon, \epsilon_{i}, \epsilon_{i j}\right),
$$

where

$$
\epsilon=\mathcal{E}(\alpha), \quad \epsilon_{i}=\left.\frac{\partial \mathcal{E}}{\partial \alpha_{i}}\right|_{\alpha}, \quad \epsilon_{i j}=\left.\frac{\partial^{2} \mathcal{E}}{\partial \alpha_{i} \partial \alpha_{j}}\right|_{\alpha} .
$$

Since (14) is integrable, the first prolongation of (14) is also integrable. Therefore for every initial condition $j_{2, \alpha} \in J_{2}(\mathfrak{g}, \mathbb{R})$ at $\alpha$, there exists a solution $\mathcal{E}: \mathfrak{g} \rightarrow \mathbb{R}$ in the neighborhood of $\alpha$ such that $j_{2}(\mathcal{E})=j_{2, \alpha}$. On the other hand, the jet $j_{2, \alpha}=\left\{\alpha, \epsilon, \epsilon_{i}, \epsilon_{i j}\right\}$ is a second order initial condition of the prolongated system if and only if $\left\{\epsilon_{i}, \epsilon_{i j}\right\}$ is a solution of the system composed by (16) and (17). Moreover the solution $\mathcal{E}$ corresponding to $j_{2, \alpha}$ is nondegenerate if and only if $\operatorname{det}\left(\epsilon_{i j}\right) \neq 0$.

Corollary 1: The canonical connection of a commutative Lie group is variational with respect to a left-invariant Lagrangian.

In that case $C_{i j}^{k}=0$ for all $i, j, k=1, \ldots, n$, and there is no obstruction to choose a nondegenerate initial condition for the system (14).

Corollary 2: If the derived Lie algebra is one dimensional, then there is no left-invariant variational principle for the canonical flow.

In that case all the solutions $\left\{x_{i}=\epsilon_{i}, x_{i j}=\epsilon_{i j}\right\}$ of the system composed by (16) and (17) are degenerate, and therefore there is no nondegenerate initial condition for the differential system (14).

Remark: The Lagrangian $E(\alpha)=K(\alpha, \alpha)$, where $K$ is the Killing form of $G$, is always a solution to the equations (12) and (13). That way we rediscover the well-known property of semisimple Lie groups: the canonical connection is variational with respect to a left-invariant Lagrangian.

Indeed, $E$ is left-invariant, it is not dependent on the $x$ coordinates in the $(x, \alpha)$ system, and (12) is satisfied. Moreover, at every $\alpha \in \mathfrak{g}$ and $a \in \mathfrak{g}$ we have 


$$
[a, \alpha] \frac{\partial E}{\partial \alpha}=\left.\frac{d}{\mathrm{~d} t}\right|_{t=0} K(\alpha+t[a, \alpha] ; \alpha+t[a, \alpha])=K([a, \alpha], \alpha)+K(\alpha,[a, \alpha])=0
$$

and Eq. (13) is satisfied.

\section{SOLUTION OF THE GROUP-INVARIANT INVERSE PROBLEM IN DIMENSIONS UP TO FOUR}

The variationality of the geodesic flow of the canonical connection of Lie groups was studied in Refs. 9 and 2, where the inverse problem for Lie groups up to dimension 4 were solved. Here we determine if there exits group-invariant variational principle for canonical geodesic flows. Theorem 3 formulates a necessary and sufficient condition for the Lie algebra so that the canonical flow is variational with respect to a group-invariant Lagrangian.

Jacobson in Ref. 6 gives the classification of Lie algebras of dimension 3 or less. The classification of Lie algebras of dimensions 4 and 5 can be found in Ref. 8 .

\section{A. Two-dimensional Lie groups}

There are, up to isomorphism, two algebras distinguished according to whether or not [,] is trivial or not. In the former case we have the Abelian Lie algebra, and according to Corollary 1 it is variational with respect to a $G$-invariant Lagrangian.

The latter one is the Lie algebra of the affine group of the line. Up to isomorphism the Lie algebra of the affine group of the line is the only non-Abelian two-dimensional Lie algebra. A representation of the group $G$ is given by $g=\left(\begin{array}{ll}x & y \\ 0 & 1\end{array}\right)$ where $x \neq 0$. The corresponding Lie algebra is

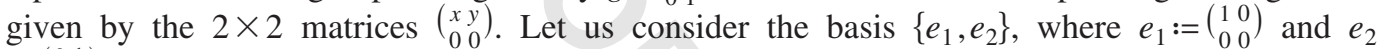
$:=\left(\begin{array}{ll}0 & 1 \\ 0 & 0\end{array}\right)$. Then $\left[e_{1}, e_{2}\right]=e_{2}$, i.e., the nonzero structure constants are $C_{12}^{2}=-C_{21}^{2}=1$. If $\left(a_{1}, a_{2}\right)$ and $\left(\alpha_{1}, \alpha_{2}\right)$ denote the coordinates of $a$ and $\alpha$ with respect to this basis, then Eq. (13) is [a, $\left.\alpha\right]$ $=\left(a_{1} \alpha_{2}-a_{2} \alpha_{1}\right) e_{2}$ which gives $\partial E / \partial \alpha_{2}=0$. Since any solution of the prolongations is nonregular we conclude that there is no invariant variational principle for the canonical connection of the affine group of the line.

\section{B. Three-dimensional Lie groups}

In Ref. 9, Thompson proved that all the canonical connections on Lie groups of dimension 3 have variational locally geodesic equations. On the other hand, Jacobson's classification of the three-dimensional Lie algebras depends primarily on the dimension of the first derived algebra $g^{(1)}$ where $g$ is the original algebra. We have the following possibilities.

If $\operatorname{dim}\left(g^{(1)}\right)=0$, then $g$ is Abelian and, according to Corollary 1 , the canonical connection of a commutative Lie group is variational with respect to a left-invariant Lagrangian. If $\operatorname{dim}\left(g^{(1)}\right)=3$, then $g$ is simple and we have $g=s \ell(2, \mathrm{R})$ or $g=s o(3)$. In both cases the Killing form provides a metric and so the connections are variational.

If $\operatorname{dim}\left(g^{(1)}\right)=1$ there are, up to isomorphism, two algebras distinguished according to whether $g^{(1)}$ lies inside the center of $g$. In the former case $g$ may be realized as the Lie algebra of the group of matrices of the form $\left[\begin{array}{lll}1 & x & y \\ 0 & 1 & z \\ 0 & 0 & 1\end{array}\right],(x, y, z \in \mathbb{R})$ and $\mathfrak{g}$ is the Heisenberg algebra. Its canonical connection is a flat connection and so it is variational. The latter case $g$ is isomorphic to the Lie algebra of the group of nonsingular $2 \times 2$ upper triangular matrices and so it is again variational.

Let us consider the Heisenberg group. A basis of the Lie algebra is given by $\left\{e_{1}, e_{2}, e_{3}\right\}$, where

$$
e_{1}:=\left(\begin{array}{lll}
0 & 1 & 0 \\
0 & 0 & 0 \\
0 & 0 & 0
\end{array}\right), \quad e_{2}:=\left(\begin{array}{lll}
0 & 0 & 1 \\
0 & 0 & 0 \\
0 & 0 & 0
\end{array}\right), \quad e_{3}:=\left(\begin{array}{lll}
0 & 0 & 0 \\
0 & 0 & 1 \\
0 & 0 & 0
\end{array}\right) .
$$

If $\left(a_{1}, a_{2}, a_{3}\right)$ and $\left(\alpha_{1}, \alpha_{2}, \alpha_{3}\right)$ denotes the coordinates of $a$ and $\alpha$ with respect to this basis, then 


$$
\left(a_{1} \alpha_{3}-a_{3} \alpha_{1}\right) \frac{\partial E}{\partial \alpha_{2}}=0
$$

and Eq. (13) gives $\partial E / \partial \alpha_{2}=0$. Since any solution of the prolongations is nonregular we conclude that there is no invariant variational principle for the canonical connection of the Heisenberg group.

Next we consider the Lie group of $2 \times 2$ upper triangular matrices $\left(\begin{array}{c}x \\ x \\ 0\end{array}\right)$ where $x z \neq 0$. Here $\left\{e_{1}, e_{2}, e_{3}\right\}$ is a basis of the Lie algebra, where

$$
e_{1}:=\left(\begin{array}{ll}
1 & 0 \\
0 & 0
\end{array}\right), \quad e_{2}:=\left(\begin{array}{ll}
0 & 1 \\
0 & 0
\end{array}\right), \quad e_{3}:=\left(\begin{array}{ll}
0 & 0 \\
0 & 1
\end{array}\right) .
$$

If $\left(a_{1}, a_{2}, a_{3}\right)$ and $\left(\alpha_{1}, \alpha_{2}, \alpha_{3}\right)$ are the coordinates of $a$ and $\alpha$, then

$$
\left(a_{1} \alpha_{2}+a_{2} \alpha_{3}-a_{1} \alpha_{2}-a_{3} \alpha_{3}\right) \frac{\partial E}{\partial \alpha_{2}}=0
$$

and Eq. (14) gives again $\partial E / \partial \alpha_{2}=0$. Therefore any solution will be nonregular. Consequently there is no invariant variational principle in this case.

\section{Four-dimensional Lie groups}

There are 12 classes of Lie algebras in dimension 4 denoted in Ref. 8 as $A_{4, i}, i=1, \ldots, 12$. The generators of the algebras are listed as $\left\{e_{1}, e_{2}, e_{3}, e_{4}\right\}$ and in each case we list the nonzero Lie brackets. Several of the Lie algebras have parameters denoted by $a$ or $b$ or both. The generic solution of the linear system (16) at a point $\alpha=\alpha_{i} e_{i}$ will be denoted by $\epsilon:=\left\{\epsilon_{i}, \epsilon_{i j}\right\}$.

In Ref. 2, Thompson and his co-workers computed all the canonical connections on Lie groups of dimension 4 and determined whether the connection is variational or not. He has shown that the Lie groups with Lie algebras $A_{4,7}, A_{4,9 b}$, and $A_{4,11 a}$, where the brackets are defined as

$$
\begin{gathered}
A_{4,7}, \quad\left[e_{2}, e_{3}\right]=e_{1}, \quad\left[e_{1}, e_{4}\right]=2 e_{1}, \quad\left[e_{2}, e_{4}\right]=e_{2}, \quad\left[e_{3}, e_{4}\right]=e_{2}+e_{3}, \\
A_{4,9 b}, \quad\left[e_{2}, e_{3}\right]=e_{1}, \quad\left[e_{1}, e_{4}\right]=(1+b) e_{1}, \quad\left[e_{2}, e_{4}\right]=e_{2}, \quad\left[e_{3}, e_{4}\right]=b e_{3}, \\
A_{4,11 a}, \quad\left[e_{2}, e_{3}\right]=e_{1}, \quad\left[e_{1}, e_{4}\right]=2 a e_{1}, \quad\left[e_{2}, e_{4}\right]=a e_{2}-e_{3}, \quad\left[e_{3}, e_{4}\right]=e_{2}+a e_{3},
\end{gathered}
$$

$-1<b<1$ and $0<a$, are not variational.

$A_{4,2 a}$, the brackets are

$$
\left[e_{1}, e_{4}\right]=a e_{1}, \quad\left[e_{2}, e_{4}\right]=e_{2}, \quad\left[e_{3}, e_{4}\right]=e_{2}+e_{3}, \quad a \neq 0 .
$$

Equation (16) leads to a solution $\left\{\epsilon_{i}, \epsilon_{i j}\right\}$, where $\epsilon_{i j}=0$ for every $(i, j) \neq(4,4)$. Therefore the condition (13) entails that the matrix $\epsilon_{i j}$ is singular, whatever is the value of $a$. Therefore the canonical flow is variational, but there is no left-invariant regular Lagrangian for its geodesics.

In the case of the Lie groups with Lie algebras $A_{4,1}, A_{4,3}, A_{4,4}, A_{4,9}$, and $A_{4,12}$, the nontrivial brackets of the algebras are determined as

$$
\begin{gathered}
A_{4,1}, \quad\left[e_{2}, e_{4}\right]=e_{1}, \quad\left[e_{3}, e_{4}\right]=e_{2}, \\
A_{4,3}, \quad\left[e_{1}, e_{4}\right]=e_{1}, \quad\left[e_{3}, e_{4}\right]=e_{2}, \\
A_{4,4}, \quad\left[e_{1}, e_{4}\right]=e_{1}, \quad\left[e_{2}, e_{4}\right]=e_{1}+e_{2}, \quad\left[e_{3}, e_{4}\right]=e_{2}+e_{3}, \\
A_{4,9}, \quad\left[e_{2}, e_{3}\right]=e_{1}, \quad\left[e_{1}, e_{4}\right]=2 e_{1}, \quad\left[e_{2}, e_{4}\right]=e_{2}, \quad\left[e_{3}, e_{4}\right]=e_{3},
\end{gathered}
$$




$$
A_{4,12}, \quad\left[e_{1}, e_{3}\right]=e_{1}, \quad\left[e_{2}, e_{3}\right]=e_{2}, \quad\left[e_{1}, e_{4}\right]=-e_{2}, \quad\left[e_{2}, e_{4}\right]=e_{1} .
$$

We can find that the general solution of Eq. (16) can be described as follows: $\epsilon_{33}, \epsilon_{34}, \epsilon_{44}$ are arbitrary numbers and the other components are all zero. Therefore

$$
\epsilon_{i j}=\left(\begin{array}{cccc}
0 & 0 & 0 & 0 \\
0 & 0 & 0 & 0 \\
0 & 0 & \epsilon_{33} & \epsilon_{34} \\
0 & 0 & \epsilon_{34} & \epsilon_{44}
\end{array}\right) .
$$

Since $\epsilon_{i j}$ is singular, there is no left-invariant regular Lagrangian for the geodesics of the canonical connection. The situation is analogous in the following.

$\mathbf{A}_{4,5 \mathbf{a}}$, the Lie algebra relations are

$$
\left[e_{1}, e_{4}\right]=e_{1}, \quad\left[e_{2}, e_{4}\right]=a e_{2}, \quad\left[e_{3}, e_{4}\right]=b e_{3} .
$$

Depending on the value of $a$ and $b$ we have four cases,

$$
\begin{aligned}
& \text { if } a=0, \quad b=0 \text {, then } \epsilon_{i j}=\left(\begin{array}{cccc}
0 & 0 & 0 & 0 \\
0 & \epsilon_{22} & \epsilon_{23} & \epsilon_{24} \\
0 & \epsilon_{23} & \epsilon_{33} & \epsilon_{34} \\
0 & \epsilon_{24} & \epsilon_{34} & \epsilon_{44}
\end{array}\right), \\
& \text { if } a=0, \quad b \neq 0, \text { then } \epsilon_{i j}=\left(\begin{array}{cccc}
0 & 0 & 0 & 0 \\
0 & \epsilon_{22} & 0 & \epsilon_{24} \\
0 & 0 & 0 & 0 \\
0 & \epsilon_{24} & 0 & \epsilon_{44}
\end{array}\right), \\
& \text { if } a \neq 0, \quad b=0, \text { then } \epsilon_{i j}=\left(\begin{array}{cccc}
0 & 0 & 0 & 0 \\
0 & 0 & 0 & 0 \\
0 & 0 & \epsilon_{33} & \epsilon_{34} \\
0 & 0 & \epsilon_{34} & \epsilon_{44}
\end{array}\right), \\
& \text { if } a \neq 0, \quad b \neq 0 \text {, then } \epsilon_{i j}=\left(\begin{array}{cccc}
0 & 0 & 0 & 0 \\
0 & 0 & 0 & 0 \\
0 & 0 & 0 & 0 \\
0 & 0 & 0 & \epsilon_{44}
\end{array}\right) .
\end{aligned}
$$

In all three cases the Lagrangian must be nonregular, and therefore there is no left-invariant regular Lagrangian for the geodesics of the canonical connection.

$\mathbf{A}_{\mathbf{4}, 6 \mathbf{b}}$, the Lie algebra relations are

$$
\left[e_{1}, e_{4}\right]=a e_{1}, \quad\left[e_{2}, e_{4}\right]=b e_{2}-e_{3}, \quad\left[e_{3}, e_{4}\right]=b e_{3},
$$

with $a \neq 0, b \geqslant 0$. Depending on the value of $a$ and $b$ we have two cases,

$$
\text { if } b \neq 0 \text {, then } \epsilon_{i j}=\left(\begin{array}{cccc}
0 & 0 & 0 & 0 \\
0 & 0 & 0 & 0 \\
0 & 0 & 0 & 0 \\
0 & 0 & 0 & \epsilon_{44}
\end{array}\right) \text {, }
$$




$$
\text { if } b=0 \text {, then } \epsilon_{i j}=\left(\begin{array}{cccc}
0 & 0 & 0 & 0 \\
0 & \epsilon_{22} & 0 & \epsilon_{24} \\
0 & 0 & 0 & 0 \\
0 & \epsilon_{24} & 0 & \epsilon_{44}
\end{array}\right) \text {. }
$$

In both cases the Lagrangian must be degenerate and therefore there is no left-invariant regular Lagrangian for the geodesics of the canonical connection.

$\mathbf{A}_{\mathbf{4}, \mathbf{8}}$, the Lie algebra relations are

$$
\left[e_{2}, e_{3}\right]=e_{1}, \quad\left[e_{2}, e_{4}\right]=e_{2}, \quad\left[e_{3}, e_{4}\right]=-e_{3} \text {. }
$$

$\epsilon_{3}, \epsilon_{4}, \epsilon_{3,2}, \epsilon_{4,1}, \epsilon_{4,4}$ are free and the matrix $\left(\epsilon_{i j}\right)$ is

$$
\left(\begin{array}{cccc}
\frac{\alpha_{4}^{2}\left(\alpha_{2} \epsilon_{32}-\epsilon_{3}\right)}{\alpha_{2}^{2} \alpha_{3}} & -\frac{\alpha_{4}\left(\alpha_{2} \epsilon_{23}-\epsilon_{3}\right)}{\alpha_{2}^{2}} & -\frac{\alpha_{4}\left(\alpha_{2} \epsilon_{32}-\epsilon_{3}\right)}{\alpha_{2} \alpha_{3}} & \epsilon_{14} \\
-\frac{\alpha_{4}\left(\alpha_{2} \epsilon_{23}-\epsilon_{3}\right)}{\alpha_{2}^{2}} & \frac{\alpha_{3}\left(\alpha_{2} \epsilon_{32}-\epsilon_{3}\right)}{\alpha_{2}^{2}} & \epsilon_{23} & -\frac{\alpha_{3}\left(\alpha_{2} \epsilon_{14}+\epsilon_{3}\right)}{\alpha_{2} \alpha_{4}} \\
-\frac{\alpha_{4}\left(\alpha_{2} \epsilon_{32}-\epsilon_{3}\right)}{\alpha_{2} \alpha_{3}} & \epsilon_{23} & \frac{\alpha_{2} \epsilon_{23}-\epsilon_{3}}{\alpha_{3}} & -\frac{\alpha_{2} \epsilon_{41}+\epsilon_{3}}{\alpha_{4}} \\
\epsilon_{14} & -\frac{\alpha_{3}\left(\alpha_{2} \epsilon_{14}+\epsilon_{3}\right)}{\alpha_{2} \alpha_{4}} & -\frac{\alpha_{2} \epsilon_{41}+\epsilon_{3}}{\alpha_{4}} & \epsilon_{44}
\end{array}\right)
$$

In Ref. 9 Thompson showed that $A_{4,8}$ admits a bi-invariant metric. In order to give a regular initial condition we can choose the free components as

$$
\epsilon_{3}:=\alpha_{2} \epsilon_{32}, \quad \epsilon_{4,1}:=-\epsilon_{23}
$$

$\epsilon_{4}, \epsilon_{4,4}$ arbitrary, $\epsilon_{3,2}$ arbitrary nonzero and compute the other components. Then we obtain a solution $\epsilon=\left\{\epsilon_{i}, \epsilon_{i j}\right\}$ of the system (16),

$$
\left(\begin{array}{cccc}
0 & 0 & 0 & -\epsilon_{23} \\
0 & 0 & \epsilon_{23} & 0 \\
0 & \epsilon_{23} & 0 & 0 \\
-\epsilon_{23} & 0 & 0 & \epsilon_{44}
\end{array}\right)
$$

where $\operatorname{det}\left(\epsilon_{i j}\right)=\left(\epsilon_{23}\right)^{4} \neq 0$. According to Theorem 3 there exists an invariant regular Lagrangian generating the canonical geodesic flow.

$\mathbf{A}_{\mathbf{4}, 10}$, the Lie algebra relations are

$$
\left[e_{2}, e_{3}\right]=e_{1}, \quad\left[e_{2}, e_{4}\right]=-e_{3}, \quad\left[e_{3}, e_{4}\right]=e_{2}
$$

The $\epsilon_{3}, \epsilon_{4}, \epsilon_{3,3}, \epsilon_{4,2}$, and $\epsilon_{4,4}$ are free components and the matrix $\left(\epsilon_{i j}\right)$ is 


$$
\left(\begin{array}{cccc}
\frac{\alpha_{4}^{2}\left(\alpha_{3} \epsilon_{33}-\epsilon_{3}\right)}{\alpha_{3}^{3}} & \frac{\alpha_{2} \alpha_{4}\left(\alpha_{3} \epsilon_{33}-\epsilon_{3}\right)}{\alpha_{3}^{3}} & \frac{\alpha_{4}\left(\alpha_{3} \epsilon_{33}-\epsilon_{3}\right)}{\alpha_{3}^{2}} & \frac{\alpha_{4} \alpha_{3} \epsilon_{24}+\alpha_{2} \epsilon_{3}}{\alpha_{2} \alpha_{3}} \\
\frac{\alpha_{2} \alpha_{4}\left(\alpha_{3} \epsilon_{33}-\epsilon_{3}\right)}{\alpha_{3}^{3}} & \frac{\alpha_{2}^{2} \alpha_{3} \epsilon_{33}+\left(\alpha_{3}^{2}-\alpha_{2}^{2}\right) \epsilon_{3}}{\alpha_{3}^{3}} & \frac{\alpha_{2}\left(\alpha_{3} \epsilon_{33}-\epsilon_{3}\right)}{\alpha_{3}^{2}} & \epsilon_{4,2} \\
\frac{\alpha_{4}\left(\alpha_{3} \epsilon_{33}-\epsilon_{3}\right)}{\alpha_{3}^{2}} & \frac{\alpha_{2}\left(\alpha_{3} \epsilon_{33}-\epsilon_{3}\right)}{\alpha_{3}^{2}} & \epsilon_{33} & \frac{\alpha_{3} \epsilon_{24}}{\alpha_{2}} \\
\frac{\alpha_{4} \alpha_{3} \epsilon_{24}+\alpha_{2} \epsilon_{3}}{\alpha_{2} \alpha_{3}} & \epsilon_{24} & \frac{\alpha_{3} \epsilon_{24}}{\alpha_{2}} & \epsilon_{44}
\end{array}\right)
$$

A nondegenerate initial condition can be chosen as follows:

$$
\epsilon_{3}:=x_{3} \epsilon_{33}, \quad \epsilon_{4,2}:=0
$$

$\epsilon_{4}, \epsilon_{4,1}, \epsilon_{4,4}$ arbitrary, $\epsilon_{3,2}$ arbitrary nonzero and the other components zero. We have

$$
\left(\begin{array}{cccc}
0 & 0 & 0 & \epsilon_{33} \\
0 & \epsilon_{33} & 0 & 0 \\
0 & 0 & \epsilon_{33} & 0 \\
\epsilon_{33} & 0 & 0 & \epsilon_{44}
\end{array}\right)
$$

and $\operatorname{det}\left(\epsilon_{i j}\right)=-\left(\epsilon_{33}\right)^{4} \neq 0$. As in the previous case, Theorem 3 guarantees the existence of an invariant regular Lagrangian generating the canonical geodesic flow.

We remark that in Ref. 9 Thompson has already shown that a Lie group with $A_{4,8}$ or $A_{4,10}$ as Lie algebra has a bi-invariant metric which generates its canonical geodesic flow.

${ }^{1}$ Cartan, E. and Schouten, J. A., "On the geometry of the group-manifold of simple and semi-simple groups," Proc. Akad. Wekensch, Amsterdam 29, 803-815 (1926).

${ }^{2}$ Granam, R., Miller, E. J., and Thompson, G., "Variationality of four-dimensional Lie group connections," J. Lie Theory 14, 395-425 (2004).

${ }^{3}$ Godbillon, C., Géométrie Différentielle et Mécanique Analytique (Hermann, Paris, 1969).

${ }^{4}$ Grifone, J., "Structure presque-tangente et connexions I, II," Ann. Inst. Fourier 22, 287-334 (1972); 22, 291-338 (1972).

${ }^{5}$ Grifone, J. and Muzsnay, Z., Variational Principles for Second-order Differential Equations (World Scientific, Singapore, 2000).

${ }^{6}$ Jacobson, N., Lie Algebras (Interscience, New York, 1962).

${ }^{7}$ Muzsnay, Z. and Thompson, G., "Inverse problem of the calculus of variations on Lie groups," Diff. Geom. Applic. 23, 257-281 (2005).

${ }^{8}$ Patera, J., Sharp, R. T., Winternitz, P., and Zassenhaus, H., "Invariants of real low dimension Lie algebras," J. Math. Phys. 17, 986-994 (1976).

${ }^{9}$ Thompson, G., "Variational connections on Lie groups," Diff. Geom. Applic. 18, 255-270 (2003). 